Supporting information for the ES\&T article

\title{
Critical factors facilitating Candidatus Nitrotoga to be prevalent nitrite-oxidizing bacteria in activated sludge
}

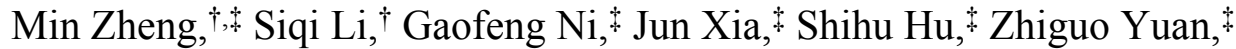 \\ Yanchen Liu, ${ }^{\dagger}, *$ Xia Huang ${ }^{\dagger}$ \\ †State Key Joint Laboratory of Environment Simulation and Pollution Control, School \\ of Environment, Tsinghua University, Beijing 100084, China \\ $¥$ Advanced Water Management Centre, The University of Queensland, St Lucia, QLD \\ 4072, Australia
}

\section{Corresponding author}

E-mail addresses: liuyc@mail.tsinghua.edu.cn (Yanchen Liu)

Number of pages: 14

Number of tables: 3

Number of figures: 9 


\section{Methods}

Sludge treatment using FNA or FA in SBRII and SBR III

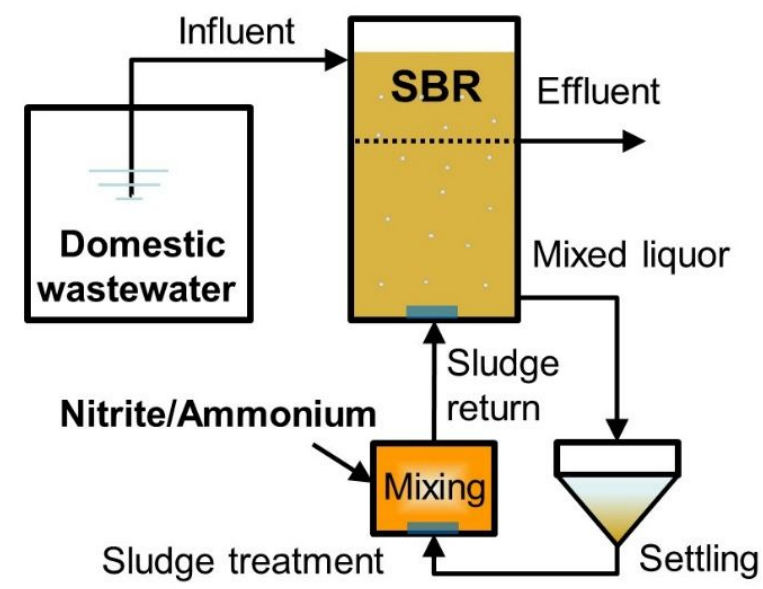

Figure S1. The schematic diagram of the lab-scale SBRs.

The daily sludge treatment using FNA was performed in SBR II from day 221 to the end of the operation. At the end of the aerobic period in each SBR operational cycle, $750 \mathrm{~mL}$ activated sludge was collected, and then gravity concentrated ten times in the settler. As such, $225 \mathrm{~mL} /$ day (three cycles per day) settled sludge was obtained. Following that, the settled sludge was transferred to the mixing unit, $18 \mathrm{mM}$ nitrite (as $\mathrm{NaNO}_{2}$ ) was added, and the mixture was stirred for 24 hours for the sludge treatment. The $\mathrm{pH}$ was controlled at 5.5 using $1 \mathrm{~N} \mathrm{HCl}$ in the mixing tank, which gave an FNA concentration of about $1.78 \mathrm{mg} \mathrm{HNO}_{2}-\mathrm{N} / \mathrm{L}$ at $23^{\circ} \mathrm{C}$. Afterward, the mixture returned back to the SBR though feeding as the influent together with the domestic wastewater. The regime repeated every day.

The daily alternating FNA and FA treatment were applied in SBR III from day 221 to the end of the operation. The sludge treatment procedure using FA was similar to that of using FNA. The difference was that in the mixing unit, $50 \mathrm{mM}$ ammonium (as $\mathrm{NH} 4 \mathrm{Cl}$ ) was added, and the $\mathrm{pH}$ was controlled at 9.0 using $1 \mathrm{~N} \mathrm{NaOH}$. As such, the FA concentration was about $153 \mathrm{mg} \mathrm{NH} \mathrm{NH}_{3} \mathrm{~N} / \mathrm{L}$ at $23^{\circ} \mathrm{C}$. 
Cloning and gene library construction.

Amplicons resulting from the Nitrotoga-specific primer pair NTG200F/NTG840R (5'-CTCGCGTTTTCGGAGCGG-3'/5'-CTAAGGAAGTCTCCTCCC-3') were cloned and sequenced. ${ }^{1}$ The amplified partial 16S rRNA genes were ligated into the plasmid vector $\mathrm{pCR}^{\mathrm{TM}} 2.1$ by using the Original TA Cloning Kit (Invitrogen by Life technologies), then transformed into One Shot ${ }^{\circledR} \mathrm{TOP} 10$ competent cells (Invitrogen by ThermoFisher Scientific). Plasmids DNA of selected transformants were obtained by picking 20 white colonies after blue/white screening from agar plates with $40 \mathrm{mg} / \mathrm{mL}$ $\mathrm{X}$-gal and $50 \mu \mathrm{g} / \mathrm{mL}$ Kanamycin. The 20 isolated, single colonies were then incubated until the culture reach stationary phase in Lysogeny broth (LB) containing $50 \mu \mathrm{g} / \mathrm{mL}$ Kanamycin separately. The constructed plasmids were extracted by using GeneJET Plasmid Miniprep Kit (Thermo Scientific). Sanger sequencing was performed at Majorbio Ltd using the universal primers T7 (5'-TAATACGACTCACTATAGGG-3') and M13 (5'-CAGGAAACAGCTATGAC-3'). ${ }^{2}$ 
Table S1. The specific primers and PCR conditions used in this study.

\begin{tabular}{|c|c|c|c|c|c|}
\hline Target gene & Primer name & Sequence $\left(5^{\prime}-3^{\prime}\right)$ & $\begin{array}{l}\text { Length of } \\
\text { amplicon }\end{array}$ & Thermal profile & Reference \\
\hline \multirow{2}{*}{$\begin{array}{l}\text { Nitrospira 16S } \\
\text { rRNA gene }\end{array}$} & NSR1113f & $\begin{array}{l}\text { CCTGCTTTCAGTTG } \\
\text { CTACCG }\end{array}$ & \multirow{2}{*}{151} & \multirow{2}{*}{$\begin{array}{l}95^{\circ} \mathrm{C} \text { for } 3 \mathrm{~min} \times 1 \\
\mathrm{cycle} ; 95^{\circ} \mathrm{C} \text { for } 30 \mathrm{~s} \text {, } \\
55^{\circ} \mathrm{C} \text { for } 30 \mathrm{~s}, 72^{\circ} \mathrm{C} \\
\text { for } 40 \mathrm{~s} \times 35 \text { cycles }\end{array}$} & \multirow{2}{*}{3} \\
\hline & NSR1264r & $\begin{array}{l}\text { GTTTGCAGCGCTTT } \\
\text { GTACCG }\end{array}$ & & & \\
\hline \multirow{2}{*}{$\begin{array}{l}\text { Ca. Nitrotoga } \\
\text { 16S rRNA gene }\end{array}$} & NTG200f & $\begin{array}{l}\text { CTCGCGTTTTCGGA } \\
\text { GCGG }\end{array}$ & \multirow{2}{*}{640} & \multirow{2}{*}{$\begin{array}{l}95^{\circ} \mathrm{C} \text { for } 3 \mathrm{~min} \times 1 \\
\text { cycle; } 95^{\circ} \mathrm{C} \text { for } 30 \mathrm{~s} \text {, } \\
56^{\circ} \mathrm{C} \text { for } 30 \mathrm{~s}, 72^{\circ} \mathrm{C} \\
\text { for } 40 \mathrm{~s} \times 35 \text { cycles }\end{array}$} & \multirow{2}{*}{1} \\
\hline & NTG840r & $\begin{array}{l}\text { CTAAGGAAGTCTCC } \\
\text { TCCC }\end{array}$ & & & \\
\hline \multirow{2}{*}{$\begin{array}{l}\text { Nitrospira nxrB } \\
\text { gene }\end{array}$} & nxrB-LRT-F & $\begin{array}{l}\text { GGCAATTGGGACG } \\
\text { GAAGAT }\end{array}$ & \multirow{2}{*}{184} & \multirow{2}{*}{$\begin{array}{l}95^{\circ} \mathrm{C} \text { for } 10 \mathrm{~min} ; \\
\left(95^{\circ} \mathrm{C} 30 \mathrm{~s} ; 60^{\circ} \mathrm{C}\right. \\
\left.30 \mathrm{~s} ; 72^{\circ} \mathrm{C} 30 \mathrm{~s}\right) 40 \\
\text { cycles }\end{array}$} & \multirow{2}{*}{4} \\
\hline & nxrB-LRT-R & $\begin{array}{l}\text { TAGGGCTTGGTCTC } \\
\text { CACGT }\end{array}$ & & & \\
\hline \multirow{2}{*}{$\begin{array}{l}\text { Comammox } \\
\text { Nitrospira } \\
\text { amoA gene }\end{array}$} & $\begin{array}{l}\text { comamoA } \\
\mathrm{AF}\end{array}$ & $\begin{array}{l}\text { AGGNGAYTGGGAY } \\
\text { TTCTGG }\end{array}$ & \multirow{2}{*}{436} & \multirow{2}{*}{$\begin{array}{l}95^{\circ} \mathrm{C} \text { for } 5 \min ;\left(95^{\circ} \mathrm{C}\right. \\
30 \mathrm{~s} ; 55^{\circ} \mathrm{C} 30 \mathrm{~s} ; 72^{\circ} \mathrm{C} \\
45 \mathrm{~s}) 35 \text { cycles; } 72^{\circ} \mathrm{C} \\
\text { for } 10 \text { min }\end{array}$} & \multirow[b]{2}{*}{5} \\
\hline & $\begin{array}{l}\text { comamoA } \\
\text { SR }\end{array}$ & $\begin{array}{l}\text { CCGVACATACATRA } \\
\text { AGCCCAT }\end{array}$ & & & \\
\hline
\end{tabular}


Table S2. The experimental conditions used in different batch experiments.

\begin{tabular}{|c|c|c|c|c|c|c|c|}
\hline No. & Sludge source & $\begin{array}{l}\text { Temp } \\
\left({ }^{\circ} \mathrm{C}\right)\end{array}$ & $\mathrm{pH}$ & $\begin{array}{l}\text { Dissolved } \\
\text { oxygen conc. } \\
\left.\text { (mg } \mathrm{O}_{2} / \mathrm{L}\right)\end{array}$ & $\begin{array}{l}\text { Initial } \\
\text { nitrite } \\
\text { conc. (mg } \\
\mathrm{N} / \mathrm{L} \text { ) } \\
\end{array}$ & $\begin{array}{l}\text { Sludge } \\
\text { treatment } \\
\text { condition }\end{array}$ & Note \\
\hline 1 & \multirow{18}{*}{$\begin{array}{l}\text { Nitrotoga- } \\
\text { dominated } \\
\text { sludge from } \\
\text { SBR III during } \\
\text { the operation } \\
\text { from day } 295 \\
\text { to day } 300 \\
\text { (ref to Figure } \\
\text { 4) }\end{array}$} & 4 & 7.2 & $>4.0$ & 25 & - & \multirow{4}{*}{$\begin{array}{l}\text { Temperat } \\
\text { ure }\end{array}$} \\
\hline 2 & & 14 & 7.2 & $>4.0$ & 25 & - & \\
\hline 3 & & 22 & 7.2 & $>4.0$ & 25 & - & \\
\hline 4 & & 30 & 7.2 & $>4.0$ & 25 & - & \\
\hline 5 & & 22 & 5.5 & $>4.0$ & 25 & - & \multirow{7}{*}{$\mathrm{pH}$} \\
\hline 6 & & 22 & 6.0 & $>4.0$ & 25 & - & \\
\hline 7 & & 22 & 6.5 & $>4.0$ & 25 & - & \\
\hline 8 & & 22 & 7.0 & $>4.0$ & 25 & - & \\
\hline 9 & & 22 & 7.5 & $>4.0$ & 25 & - & \\
\hline 10 & & 22 & 8.5 & $>4.0$ & 25 & - & \\
\hline 11 & & 22 & 9.5 & $>4.0$ & 25 & - & \\
\hline 12 & & 22 & 7.2 & 0.25 & 25 & - & \multirow{6}{*}{$\begin{array}{l}\text { Oxygen } \\
\text { affinity }\end{array}$} \\
\hline 13 & & 22 & 7.2 & 0.55 & 25 & - & \\
\hline 14 & & 22 & 7.2 & 1.0 & 25 & - & \\
\hline 15 & & 22 & 7.2 & 2.0 & 25 & - & \\
\hline 16 & & 22 & 7.2 & 3.0 & 25 & - & \\
\hline 17 & & 22 & 7.2 & 4.0 & 25 & - & \\
\hline 18 & & 22 & 7.2 & $>4.0$ & 8.4 & - & $\begin{array}{l}\text { Nitrite } \\
\text { affinity }\end{array}$ \\
\hline 19 & \multirow{5}{*}{$\begin{array}{l}\text { Nitrospira- } \\
\text { dominated } \\
\text { sludge from } \\
\text { SBR I on day } \\
330 \text { (ref to } \\
\text { Figure 5) }\end{array}$} & 22 & 7.2 & $>4.0$ & 25 & - & \multirow{10}{*}{$\begin{array}{l}\text { Sludge } \\
\text { treatment }\end{array}$} \\
\hline 20 & & 22 & 7.2 & $>4.0$ & 25 & $\mathrm{pH} 5.5$ & \\
\hline 21 & & 22 & 7.2 & $>4.0$ & 25 & $\begin{array}{l}18 \mathrm{mM} \text { nitrite } \\
\text { at } \mathrm{pH} 5.5\end{array}$ & \\
\hline 22 & & 22 & 7.2 & $>4.0$ & 25 & $\mathrm{pH} 9.0$ & \\
\hline 23 & & 22 & 7.2 & $>4.0$ & 25 & $\begin{array}{l}50 \mathrm{mM} \text { total } \\
\text { ammonia at } \\
\mathrm{pH} 9.0\end{array}$ & \\
\hline 24 & \multirow{5}{*}{ 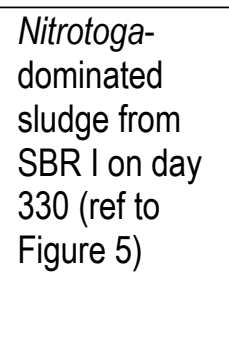 } & 22 & 7.2 & $>4.0$ & 25 & - & \\
\hline 25 & & 22 & 7.2 & $>4.0$ & 25 & pH 5.5 & \\
\hline 26 & & 22 & 7.2 & $>4.0$ & 25 & $\begin{array}{l}18 \mathrm{mM} \text { nitrite } \\
\text { at } \mathrm{pH} 5.5\end{array}$ & \\
\hline 27 & & 22 & 7.2 & $>4.0$ & 25 & pH 9.0 & \\
\hline 28 & & 22 & 7.2 & $>4.0$ & 25 & $\begin{array}{l}50 \mathrm{mM} \text { total } \\
\text { ammonia at } \\
\mathrm{pH} 9.0\end{array}$ & \\
\hline
\end{tabular}


Table S3. Binding site for the 16S rRNA genes of $\mathrm{Ca}$. Nitrotoga used to determine the matches and mismatches of NTG200f and NTG840r primers. Sites with blue font indicate matches with primer, while red indicates mismatches.

\begin{tabular}{|l|l|l|l|l|l|l|l|l|l|l|l|l|l|l|l|l|l|l|l|}
\hline & $\begin{array}{l}\text { Primer NTG 200f used in } \\
\text { this study }\end{array}$ & C & T & C & G & C & G & T & T & T & T & C & G & G & A & G & C & G & G \\
\hline Reference sequence & Accession number & & & & & & & & & & & & & & & & & & \\
\hline Ca. Nitrotoga. HW29 & KT778545.1 & C & T & C & G & C & G & T & T & T & T & C & G & G & A & G & C & G & G \\
\hline Ca. Nitrotoga. SPKER & QFXG01000001.1 & C & T & C & G & C & G & T & T & T & T & C & G & G & A & G & C & G & G \\
\hline Ca. Nitrotoga. MKT & QFXH01000001.1 & C & T & C & G & C & G & T & T & T & T & C & G & G & A & G & C & G & G \\
\hline Ca. Nitrotoga. LAW & QFXI01000001.1 & C & T & C & G & C & G & T & T & T & T & C & G & G & A & G & C & G & G \\
\hline Ca. Nitrotoga. fabula & LS423452.1 & C & T & C & G & C & G & T & T & T & T & C & G & G & A & G & C & G & G \\
\hline Ca. Nitrotoga. BS & MF555727.1 & C & T & C & G & C & G & T & T & T & T & C & G & G & A & G & C & G & G \\
\hline Ca. Nitrotoga. HAM-1 & FJ263061.1 & C & T & C & G & C & G & T & T & T & T & C & G & G & A & G & C & G & G \\
\hline Ca. Nitrotoga. CP45 & QFXJ01000059.1 & C & T & C & G & C & G & T & T & T & T & C & G & G & A & G & C & G & G \\
\hline Ca. Nitrotoga. AM1 & LC190436.1 & C & T & C & G & C & G & T & T & T & T & C & G & G & A & G & C & G & G \\
\hline Ca. Nitrotoga. arctica & DQ839562.1 & C & T & C & G & C & G & T & T & T & T & C & G & G & A & G & C & G & G \\
\hline
\end{tabular}

\begin{tabular}{|l|l|l|l|l|l|l|l|l|l|l|l|l|l|l|l|l|l|l|l|l|}
\hline & $\begin{array}{l}\text { Primer NTG 840r used in } \\
\text { this study }\end{array}$ & $\mathbf{C}$ & $\mathbf{T}$ & $\mathbf{A}$ & $\mathbf{A}$ & $\mathbf{G}$ & $\mathbf{G}$ & $\mathbf{A}$ & $\mathbf{A}$ & $\mathbf{G}$ & $\mathbf{T}$ & $\mathbf{C}$ & $\mathbf{T}$ & $\mathbf{C}$ & $\mathbf{C}$ & $\mathbf{T}$ & $\mathbf{C}$ & $\mathbf{C}$ & $\mathbf{C}$ \\
\hline Reference sequence & Accession number & & & & & & & & & & & & & & & & & & \\
\hline Ca. Nitrotoga. HW29 & KT778545.1 & C & T & A & A & G & G & A & A & G & T & C & T & C & C & T & C & C & C \\
\hline Ca. Nitrotoga. SPKER & QFXG0100001.1 & C & T & A & A & G & G & A & A & G & T & C & T & C & C & T & C & C & C \\
\hline Ca. Nitrotoga. MKT & QFXH01000001.1 & C & T & A & A & G & G & A & A & G & T & C & T & C & C & T & C & C & C \\
\hline Ca. Nitrotoga. LAW & QFX101000001.1 & C & T & A & A & G & G & A & A & G & T & C & T & C & C & T & C & C & C \\
\hline Ca. Nitrotoga. fabula & LS423452.1 & C & T & A & A & G & G & A & A & G & T & C & T & C & C & C & C & C & C \\
\hline Ca. Nitrotoga. BS & MF555727.1 & C & T & A & A & G & G & A & A & G & T & C & T & C & C & T & C & C & C \\
\hline Ca. Nitrotoga. HAM-1 & FJ263061.1 & C & T & A & A & G & G & A & A & G & T & C & T & C & C & T & C & C & C \\
\hline Ca. Nitrotoga. CP45 & QFXJ01000059.1 & C & T & A & A & G & G & A & A & G & T & C & T & C & C & T & C & C & C \\
\hline Ca. Nitrotoga. AM1 & LC190436.1 & C & T & A & A & G & G & A & A & G & T & C & T & C & C & T & C & C & C \\
\hline Ca. Nitrotoga. arctica & DQ839562.1 & C & T & A & A & G & G & A & A & G & T & C & T & C & C & T & C & C & C \\
\hline
\end{tabular}




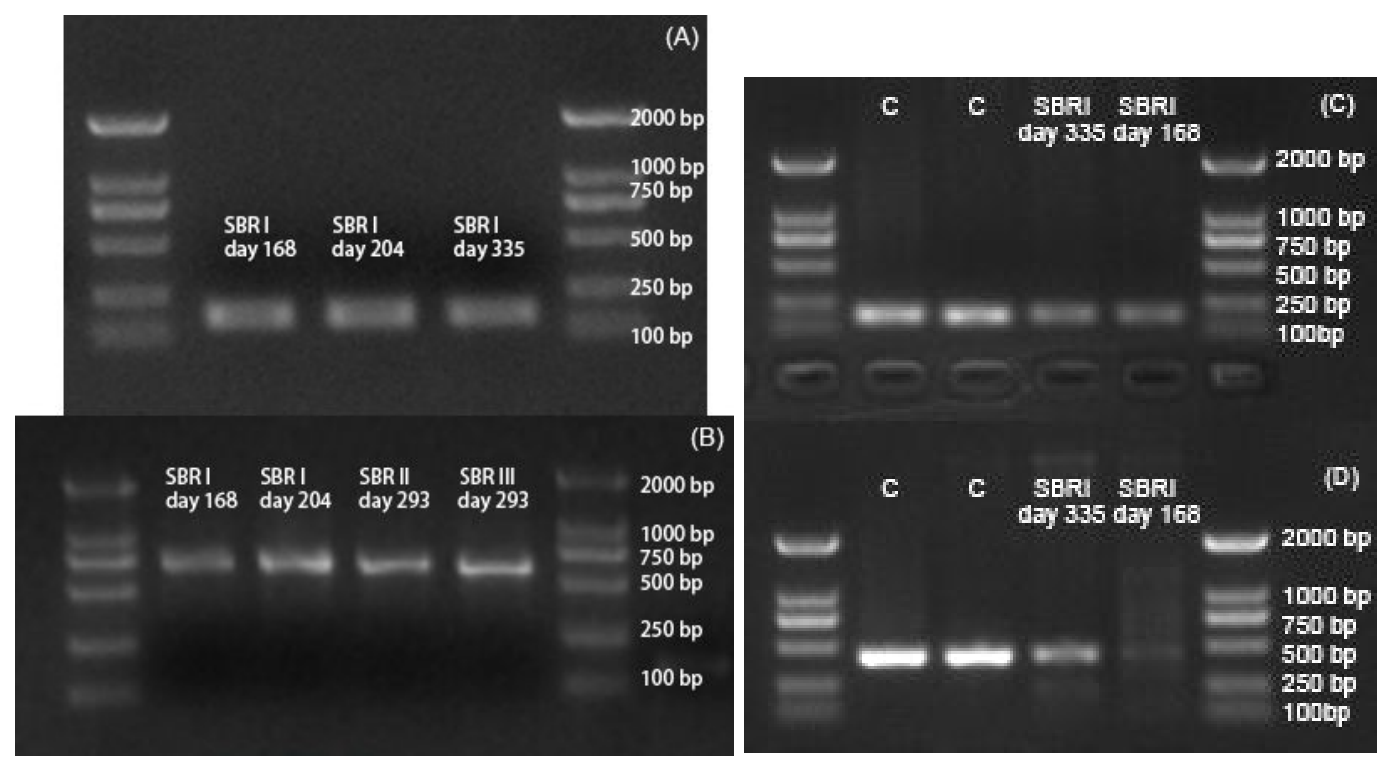

Figure S2. Agarose gel electrophoresis of PCR amplification of the Nitrospira 16S rRNA gene (A), Ca. Nitrotoga 16S rRNA gene (B), Nitrospira $n x r$ B gene (C) and comammox Nitrospira amoA gene (D). The results from figures (C) and (D) show that the $n x r \mathrm{~B}$ gene (184bp) and comammox amoA gene (436bp) are presented in the sample 'SBR I day 335', as well as two positive control (i.e., c in figure) samples, which were collected from the highly enriched comammox culture in our laboratory. ${ }^{6}$ Besides, the sample 'SBR I day 168' possibly contains the comammox Nitrospira. 


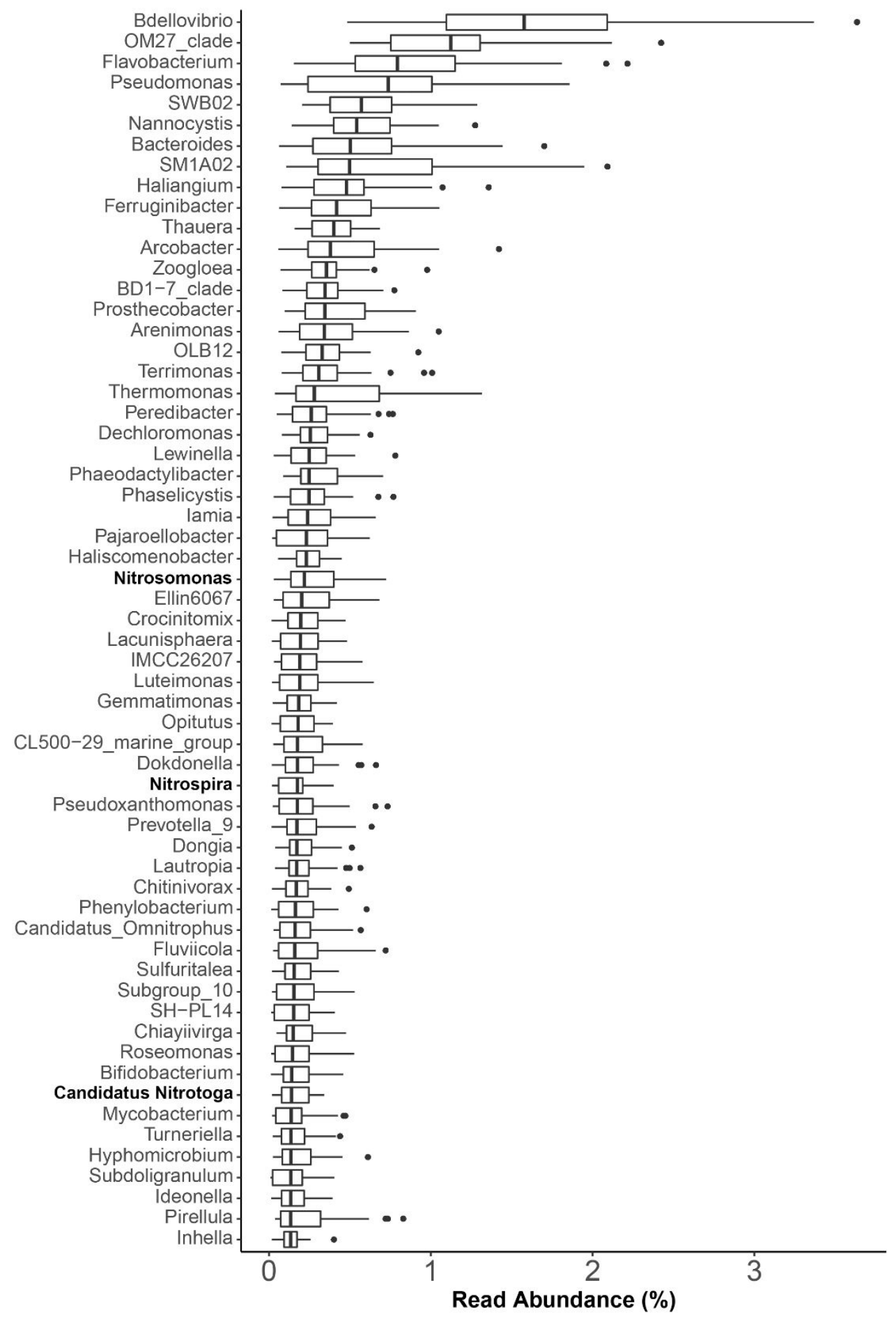

Figure S3. Box dot plot of the abundance of the top 60 genus-level ASVs (by median).

The upper and lower bounds of boxes denote the $25^{\text {th }}$ and $75^{\text {th }}$ percentiles and the lines denote the max and min values, outliers $(\bullet)$ are shown as dots. ASV labels are the lowest assigned taxonomic rank. 


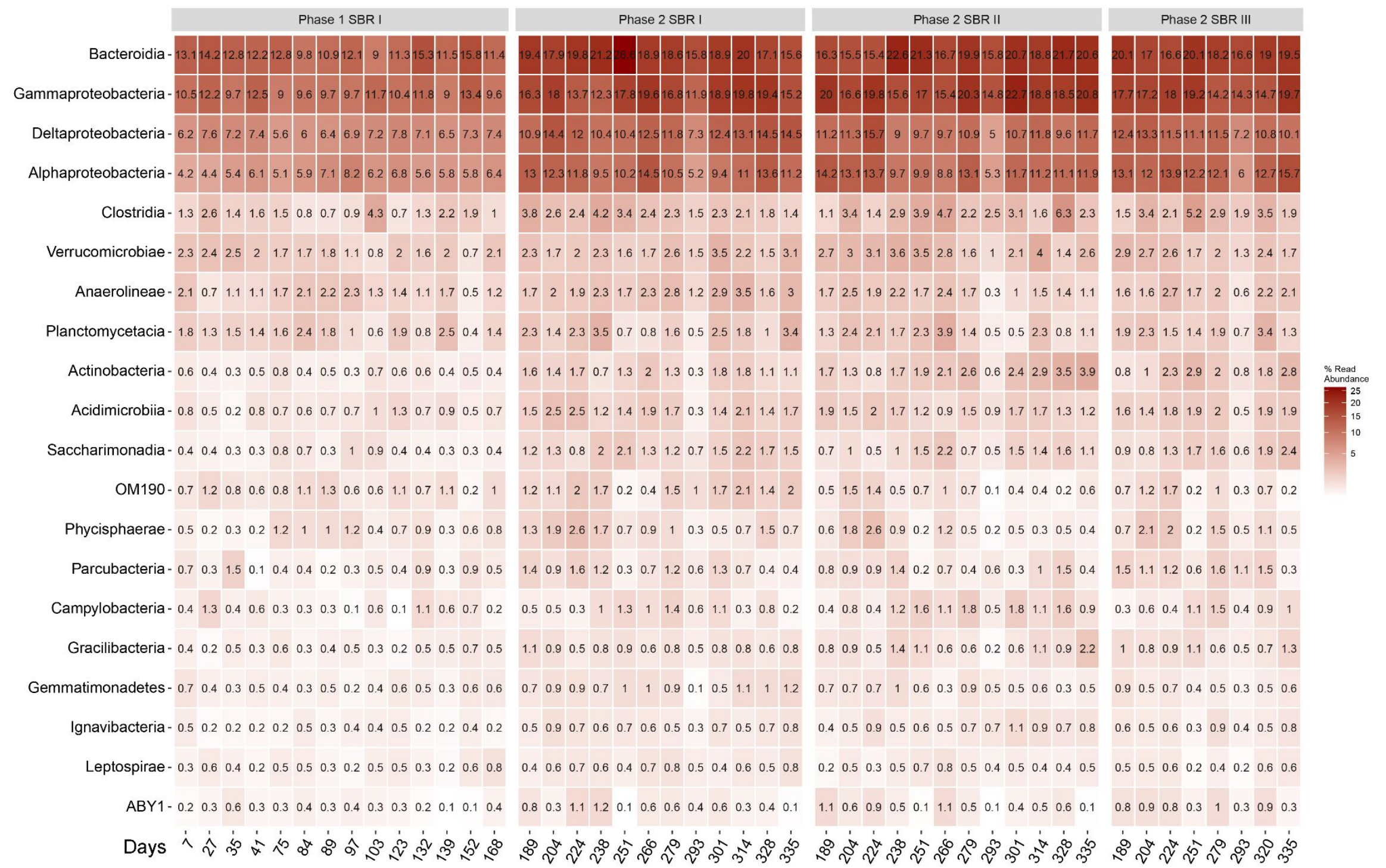

Figure S4. Heatmap plotting the microbial community (obtained from 16S rRNA gene amplicon sequencing) at a class level (top 20). 46 sludge samples in total were collected. 

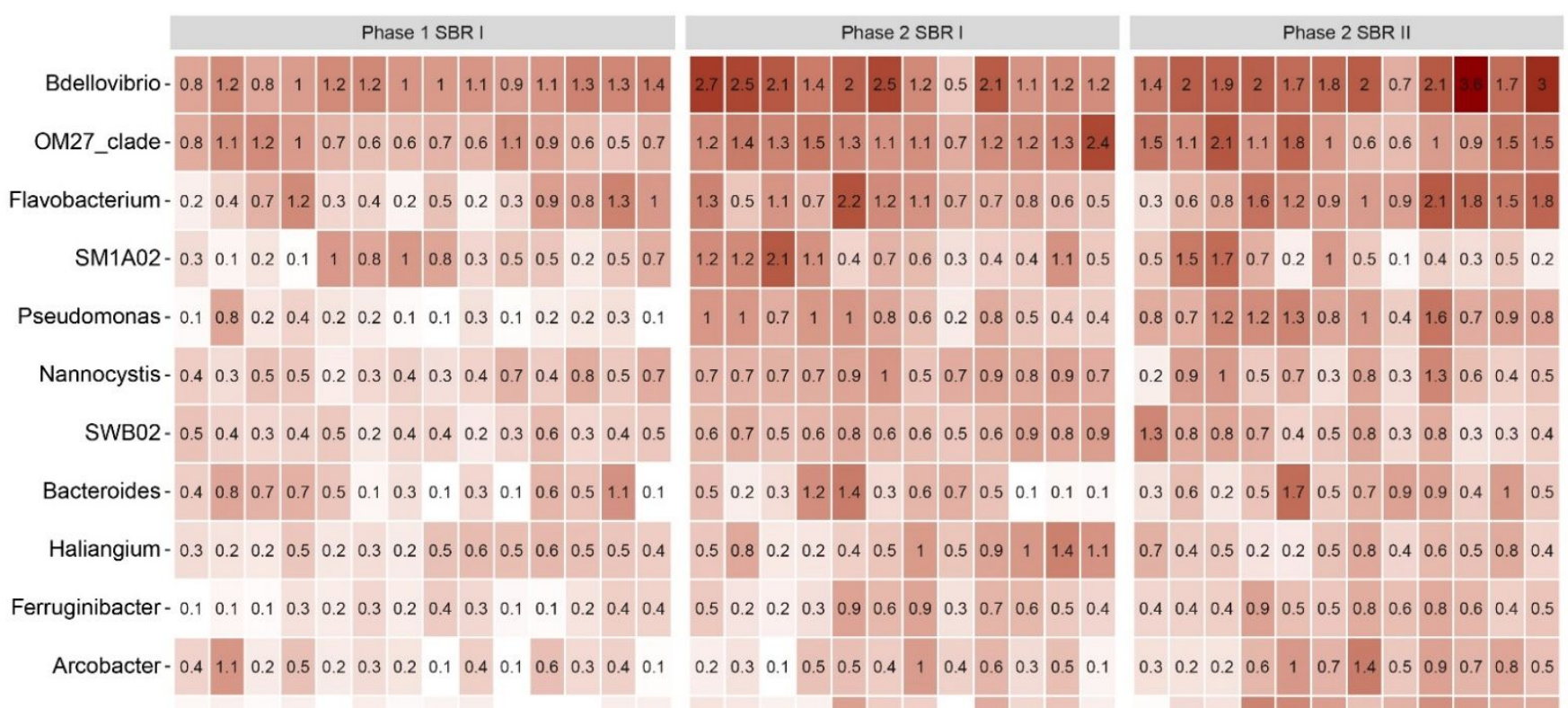

Phase 2 SBR III

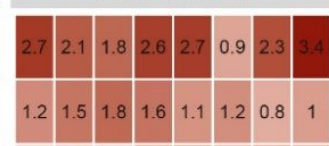

\begin{tabular}{lllllllllllllllllllll|l|l|l|l|l|l|}
1.3 & 0.5 & 1.1 & 0.7 & 2.2 & 1.2 & 1.1 & 0.7 & 0.7 & 0.8 & 0.6 & 0.5 & 0.3 & 0.6 & 0.8 & 1.6 & 1.2 & 0.9 & 1 & 0.9 & 2.1 & 1.8 & 1.5 & 1.8
\end{tabular}

$\begin{array}{lllllllll}0.5 & 0.6 & 0.6 & 0.9 & 1.1 & 0.7 & 1 & 0.9\end{array}$

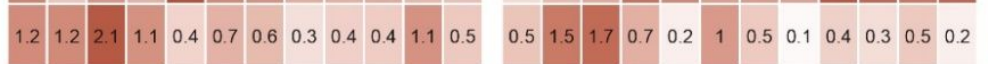

\begin{tabular}{l|l|l|l|l|l|l|l|l|l|l|l}
\hline 1 & 1 & 0.7 & 1 & 1 & 0.8 & 0.6 & 0.2 & 0.8 & 0.5 & 0.4 & 0.4
\end{tabular}

\begin{tabular}{llllll|l|l|l|l|l|l}
0.7 & 0.7 & 0.7 & 0.7 & 0.9 & 1 & 0.5 & 0.7 & 0.9 & 0.8 & 0.9 & 0.7
\end{tabular}

\begin{tabular}{llllllllll|l|l}
0.6 & 0.7 & 0.5 & 0.6 & 0.8 & 0.6 & 0.6 & 0.5 & 0.6 & 0.9 & 0.8 & 0.9
\end{tabular}

\begin{tabular}{l|l|l|l|l|l|l|l|l|l|l|l|l|l}
0.8 & 0.7 & 1.2 & 1.2 & 1.3 & 0.8 & 1 & 0.4 & 1.6 & 0.7 & 0.9 & 0.8 \\
\hline
\end{tabular}

\begin{tabular}{ll|llllllllll}
0.2 & 0.9 & 1 & 0.5 & 0.7 & 0.3 & 0.8 & 0.3 & 1.3 & 0.6 & 0.4 & 0.5
\end{tabular}

$\begin{array}{llllllllllll}1.3 & 0.8 & 0.8 & 0.7 & 0.4 & 0.5 & 0.8 & 0.3 & 0.8 & 0.3 & 0.3 & 0.4\end{array}$

\begin{tabular}{lllllllllllllllllllll|l|l|l|l}
0.5 & 0.2 & 0.3 & 1.2 & 1.4 & 0.3 & 0.6 & 0.7 & 0.5 & 0.1 & 0.1 & 0.1 & 0.3 & 0.6 & 0.2 & 0.5 & 1.7 & 0.5 & 0.7 & 0.9 & 0.9 & 0.4 & 1 & 0.5
\end{tabular}

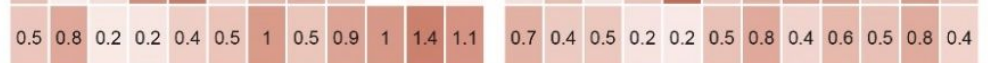

$\begin{array}{llllllllllll}0.5 & 0.2 & 0.2 & 0.3 & 0.9 & 0.6 & 0.9 & 0.3 & 0.7 & 0.6 & 0.5 & 0.4\end{array}$

$\begin{array}{lllllllllllll}0.4 & 0.4 & 0.4 & 0.9 & 0.5 & 0.5 & 0.8 & 0.6 & 0.8 & 0.6 & 0.4 & 0.5\end{array}$

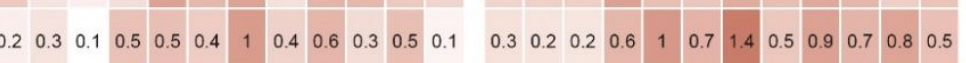

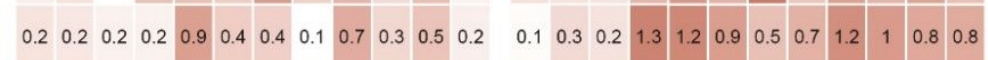

Thermomonas - $0.1 \quad 0.2 \quad 0.1 \quad 0.2 \quad 0.1 \quad 0.2 \quad 0.1 \quad 0.1 \quad 0.2 \quad 0 \quad 0.1 \quad 0 \quad 0.20 .2$

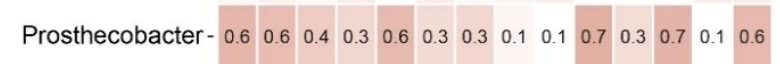

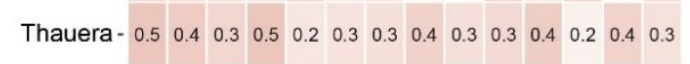

$\begin{array}{lllllllllllll}0.5 & 0.3 & 0.3 & 0.5 & 0.3 & 0.2 & 0.3 & 0.3 & 0.9 & 0.1 & 0.1 & 0.3\end{array}$

\begin{tabular}{lllllllll|l|l|l|l}
0.3 & 0.7 & 0.2 & 0.3 & 0.4 & 0.2 & 0.2 & 0.6 & 0.4 & 0.5 & 0.4 & 0.3
\end{tabular}

$\begin{array}{llllllllllll}0.5 & 0.5 & 0.7 & 0.6 & 0.7 & 0.7 & 0.3 & 0.2 & 0.1 & 0.7 & 0.2 & 0.6\end{array}$

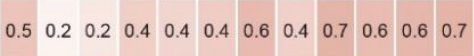

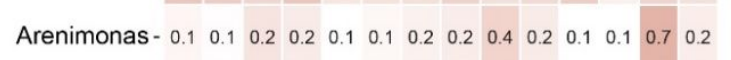

Zoogloea- $\begin{array}{llllllllllllll}0.2 & 0.4 & 0.3 & 0.3 & 0.2 & 0.3 & 0.4 & 0.3 & 0.4 & 0.2 & 0.2 & 0.2 & 0.4 & 0.3\end{array}$

BD1-7_clade- $\begin{array}{lllllllllllllll}0.3 & 0.6 & 0.4 & 0.4 & 0.1 & 0.2 & 0.3 & 0.3 & 0.2 & 0.2 & 0.4 & 0.1 & 0.2 & 0.2\end{array}$

$\begin{array}{llllllllllll}0.2 & 0.5 & 0.1 & 0.2 & 0.3 & 0.1 & 0.5 & 0.1 & 0.3 & 0.4 & 0.5 & 0.3\end{array}$

$\begin{array}{lllllllllllll}0.3 & 0.4 & 0.3 & 0.2 & 0.5 & 0.5 & 0.5 & 0.8 & 0.8 & 0.6 & 0.7 & 0.9\end{array}$

Terrimonas- $\begin{array}{llllllllllllll}0.3 & 0.1 & 0.1 & 0.2 & 0.2 & 0.3 & 0.2 & 0.2 & 0.2 & 0.4 & 0.3 & 0.2 & 0.1 & 0.1\end{array}$

$\begin{array}{llllllllllll}0.4 & 0.4 & 0.1 & 0.3 & 0.5 & 0.6 & 0.4 & 0.7 & 0.5 & 0.4 & 0.3 & 0.3\end{array}$

$\begin{array}{llllllllllll}1 & 0.3 & 0.4 & 0.3 & 0.3 & 0.5 & 0.4 & 0.5 & 0.6 & 0.5 & 0.1 & 0.5\end{array}$

\begin{tabular}{ll|llllll}
0.7 & 1.9 & 1.7 & 0.2 & 1.2 & 0.3 & 0.9 & 0.2
\end{tabular}

\begin{tabular}{lll|l|l|l|l|l|l}
0.8 & 0.7 & 1.1 & 1.9 & 1.1 & 0.2 & 0.9 & 1.2
\end{tabular}

$\begin{array}{lllllllll}0.9 & 0.5 & 0.7 & 0.4 & 0.4 & 0.4 & 0.5 & 0.1\end{array}$

\begin{tabular}{ll|l|l|l|l|l|l}
0.8 & 0.7 & 0.8 & 0.6 & 0.7 & 0.4 & 0.8 & 0.9
\end{tabular}

\begin{tabular}{lllllll|l|l|l}
0.2 & 0.6 & 0.3 & 1.3 & 1.3 & 0.8 & 1.1 & 0.3
\end{tabular}

$\begin{array}{lllllllll}0.5 & 0.5 & 0.3 & 0.3 & 0.5 & 0.2 & 0.1 & 0.1\end{array}$

\begin{tabular}{lllllll|l|l|l}
0.4 & 0.7 & 0.5 & 0.8 & 1.1 & 0.5 & 0.8 & 0.9
\end{tabular}

$\begin{array}{lllllllll}0.1 & 0.2 & 0.2 & 0.7 & 0.9 & 0.3 & 0.4 & 0.7\end{array}$ \begin{tabular}{lllllll|l|l}
0.3 & 0.2 & 0.5 & 0.8 & 0.4 & 0.4 & 0.6 & 1.2
\end{tabular}

$\begin{array}{llllllllll}0.5 & 0.4 & 0.5 & 0.2 & 0.2 & 0.2 & 0.4 & 0.2\end{array}$ $\begin{array}{llllllllll}0.6 & 0.4 & 0.2 & 0.6 & 0.4 & 0.6 & 0.3 & 0.7\end{array}$ $\begin{array}{lllllllll}0.5 & 0.5 & 0.4 & 0.8 & 0.3 & 1 & 0.5 & 0.8\end{array}$

$\begin{array}{llllllll}0.4 & 0.3 & 0.4 & 0.3 & 0.3 & 0.4 & 0.3 & 0.5\end{array}$

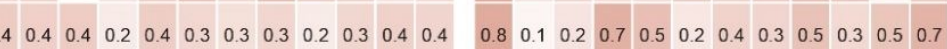
\begin{tabular}{llll|llllllll}
0.2 & 0.2 & 0.4 & 0.4 & 1 & 1 & 0.3 & 0.2 & 0.3 & 0.4 & 0.4 & 0.5
\end{tabular} \begin{tabular}{lllllllllll|l|l|l|l} 
OLB12- 0.3 & 0.1 & 0.2 & 0.1 & 0.3 & 0.4 & 0.3 & 0.4 & 0.2 & 0.4 & 0.5 & 0.3 & 0.6 & 0.3
\end{tabular}

$\begin{array}{llllllllllllllll}\text { Phaeodactylibacter }-0.7 & 0.2 & 0.1 & 0.4 & 0.3 & 0.2 & 0.2 & 0.5 & 0.5 & 0.3 & 0.5 & 0.2 & 0.2 & 0.1\end{array}$

$\begin{array}{llllllllllll}0.3 & 0.6 & 0.4 & 0.3 & 0.4 & 0.6 & 0.5 & 0.2 & 0.3 & 0.3 & 0.5 & 0.4\end{array}$ $\begin{array}{llllllllllll}0.2 & 0.4 & 0.3 & 0.2 & 0.6 & 0.4 & 0.2 & 0.2 & 0.4 & 0.5 & 0.4 & 0.4\end{array}$

$\begin{array}{llllllllllll}0.3 & 0.1 & 0.4 & 0.4 & 0.2 & 0.4 & 0.8 & 0.4 & 0.3 & 0.3 & 0.1 & 0.3\end{array}$ $\begin{array}{llllllll}0.5 & 0.2 & 0.3 & 0.7 & 0.4 & 0.2 & 0.4 & 0.7\end{array}$ $\begin{array}{lllllllll}0.2 & 0.3 & 0.4 & 0.6 & 0.6 & 0.4 & 0.4 & 0.6\end{array}$ $\begin{array}{llllllll}0.5 & 0.9 & 0.6 & 0.2 & 0.3 & 0.2 & 0.1 & 0.2\end{array}$

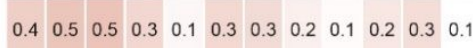
$\begin{array}{lllllllllllllllllllllll}0.2 & 0.2 & 0.2 & 0.2 & 0.2 & 0.4 & 0.5 & 0.1 & 0.3 & 0.3 & 0.2 & 0.4 & 0.2 & 0.5 & 0.1 & 0.2 & 0.2 & 0.2 & 0.2 & 0.3\end{array}$

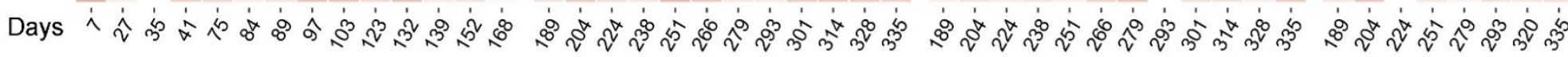

Figure S5. Heatmap plotting the microbial community (obtained from 16S rRNA gene amplicon sequencing) at a genus level (top 20). 46 sludge samples in total were collected. 


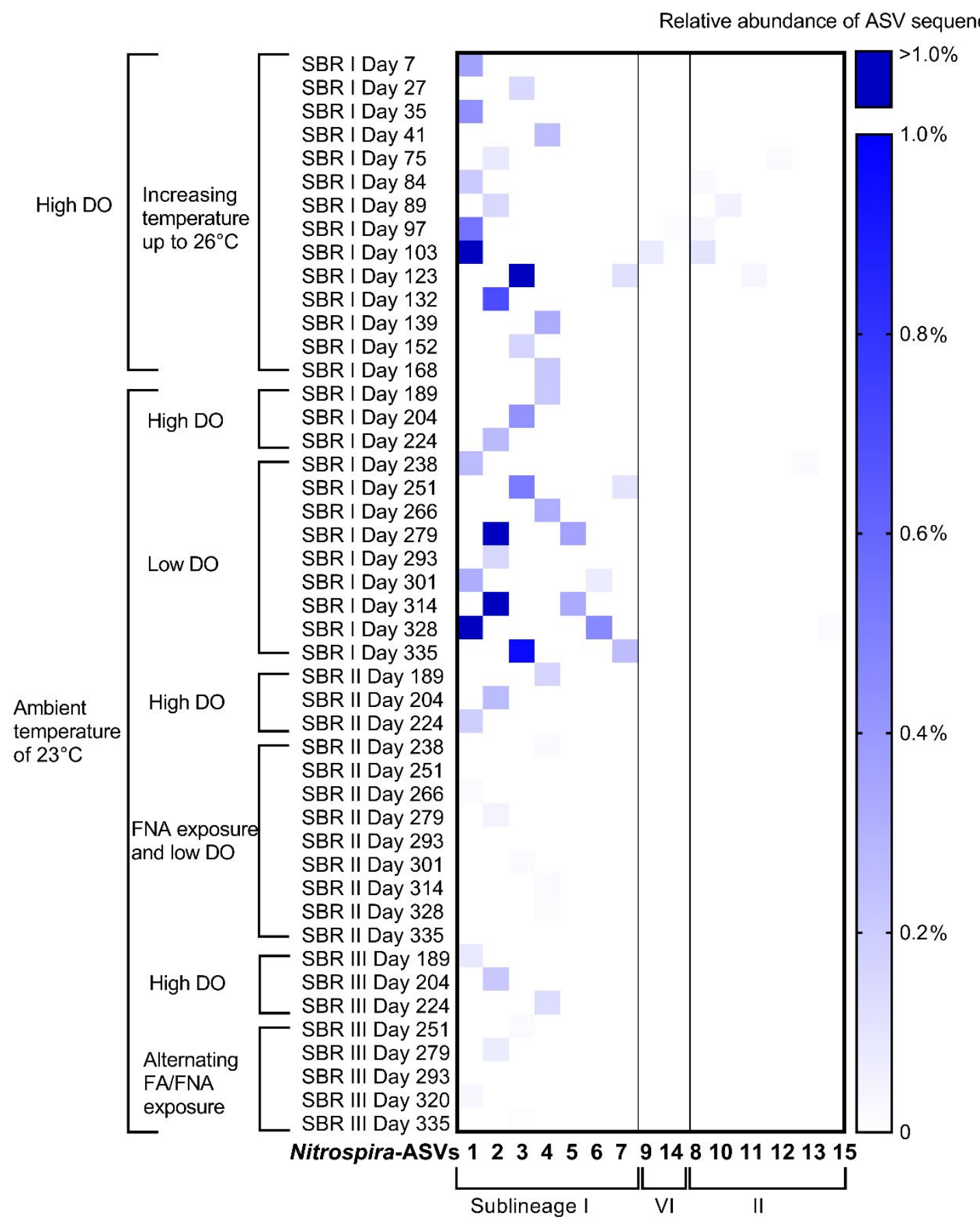

Figure S6. Heatmap plotting the relative abundance of Nitrospira-ASVs. 


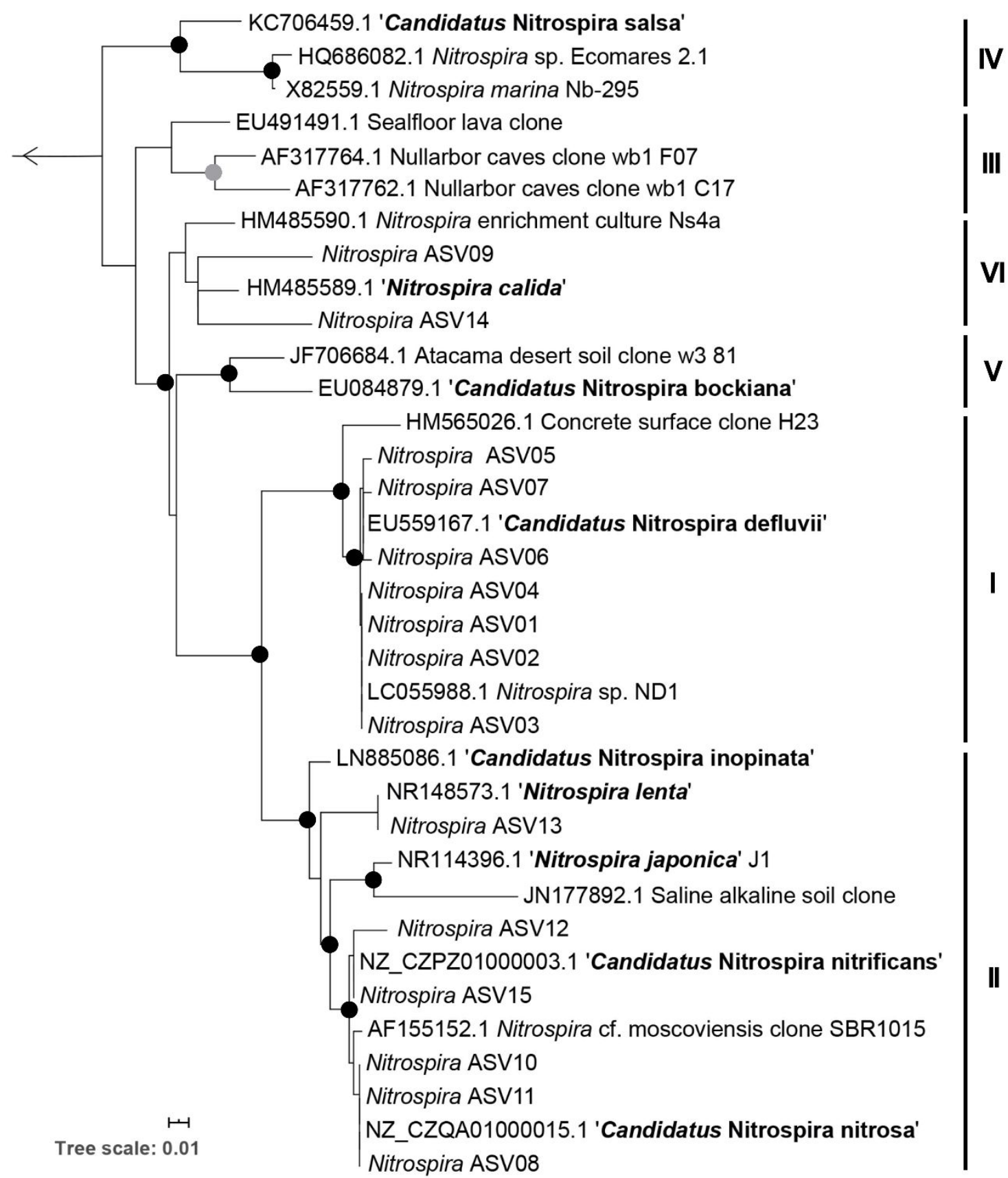

Figure S7. 16S rRNA gene maximum likelihood phylogenetic tree of ASVs affiliated with the genus Nitrospira together with public-available Nitrospira representatives. Bootstrap was set at 1,000 iterations, and Bootstrap was set at 1,000 iterations, and the grey and black circles indicate bootstrap values $>70 \%$ and $>90 \%$, respectively. Sublineage classification for the genus Nitrospira is indicated by Roman numbers on the right. Leptospirillum ferriphilum strain (AF356829.1) was used as an outgroup. 
Relative abundance of ASV sequences

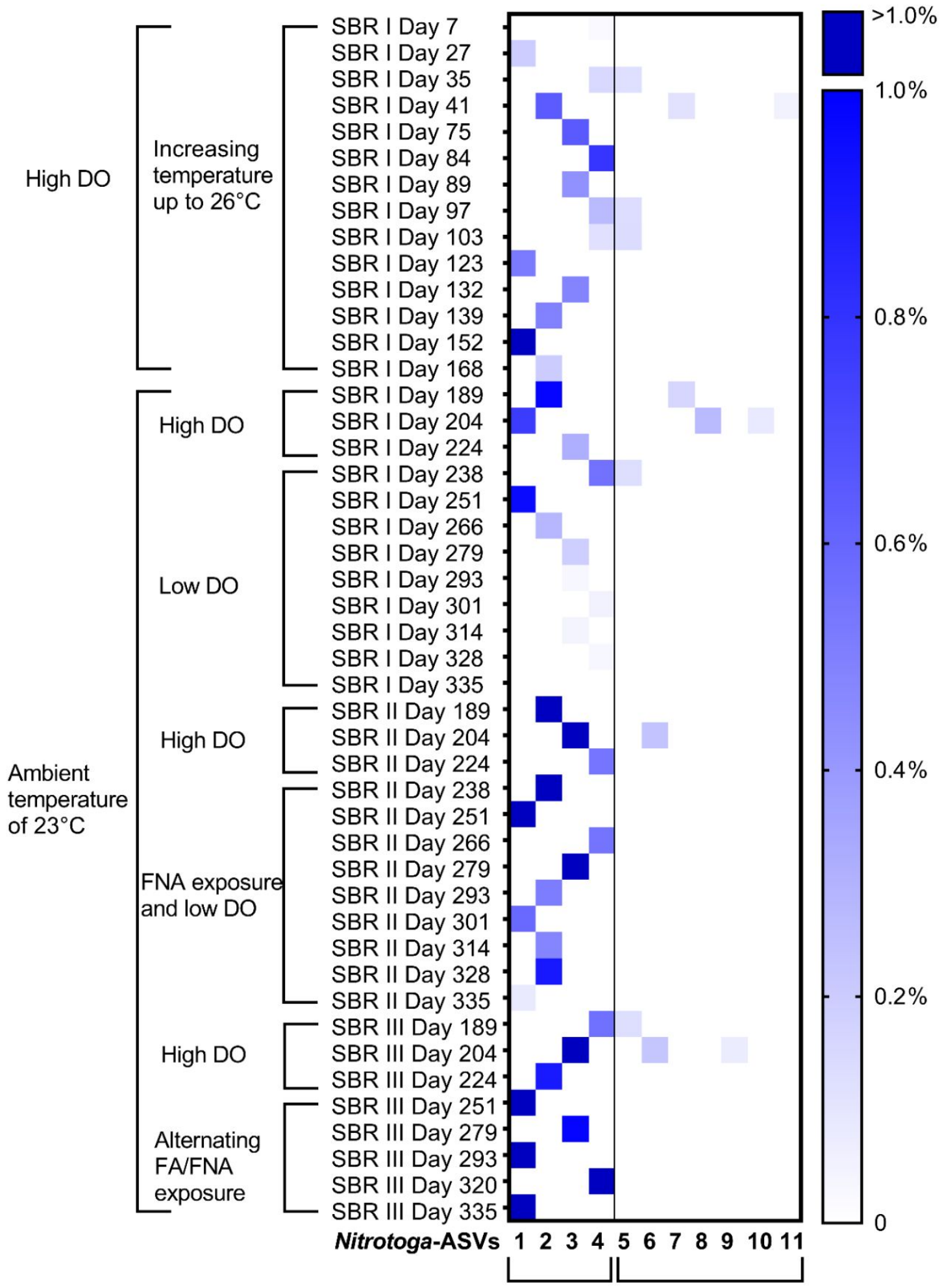

Figure S8. Heatmap plotting the relative abundance of Nitrotoga-ASVs. Nitrotoga-ASVs 1-4 were the most abundant in the dataset and are closely related to $\mathrm{Ca}$. Nitrotoga fabula. 


\section{References}

1. Alawi M, Lipski A, Sanders T, Spieck, E. (2007). Cultivation of a novel cold-adapted nitrite oxidizing betaproteobacterium from the Siberian Arctic. The ISME Journal 1 (3): 256-164.

2. Delbès C, Moletta R, Godon JJ. (2000). Monitoring of activity dynamics of an anaerobic digestor bacterial community using $16 \mathrm{~S}$ rRNA PCR-Single-Strand Conformation Polymorphism analysis (SSCP). Environ Microbiol 5: 506-515.

3. Dionisi HM, Layton AC, Harms G, Gregory IR, Robinson KG, Sayler GS. (2002). Quantification of Nitrosomonas oligotropha-like ammonia-oxidizing bacteria and Nitrospira spp. from full-scale wastewater treatment plants by competitive PCR. Appl Environ Microbiol 68 (1): 245-253.

4. Gerbl FW, Weidler GW, Wanek W, Erhardt A, Stan-Lotter H. (2014). Thaumarchaeal ammonium oxidation and evidence for a nitrogen cycle in a subsurface radioactive thermal spring in the Austrian Central Alps. Front Microbiol 5, 225.

5. Wang M, Huang G, Zhao Z, Dang C, Liu W, Zheng M. (2018). Newly designed primer pair revealed dominant and diverse comammox amoA gene in full-scale wastewater treatment plants. Bioresour Technol 270, 580-587.

6. Li J, Hua Z-S, Liu T, Wang C, Li J, Bai G, Lücker S, Jetten MSM, Zheng M, Guo J. Selective enrichment and metagenomic analysis of three novel comammox Nitrospira in a urine-fed membrane bioreactor. Submitted for publication. 